\title{
Clinical and economic benefits of continuous blood glucose monitoring devices
}

\author{
Rahmathulla Safiyul Rahman ${ }^{1 *}$, Hashim Essam Salamah², Fahad Mohammed Alshair ${ }^{2}$, \\ Abdullah Abdulhakim Alsawadi ${ }^{2}$, Oday Mohammed Alghamdi ${ }^{2}$, Ahmed Bader Alalasi ${ }^{2}$, \\ Ahmed Mohammed Mufarrihi ${ }^{2}$, Ali Omar Alalawi ${ }^{3}$, Olayan Mohammed Alzahrani ${ }^{4}$, \\ Hamoud Obaid Alanazi ${ }^{5}$, Ahmed Ali Alghamdi ${ }^{6}$, Amaar Mohammed Saleh Alsyamy ${ }^{7}$
}

\author{
${ }^{1}$ Department of Internal Medicine, Sameera Medical Center, Jeddah, Saudi Arabia \\ ${ }^{2}$ College of Medicine, King Abdulaziz University, Jeddah, Saudi Arabia \\ ${ }^{3}$ Department of Internal Medicine, South Qunfundah Hospital, Qunfundah, Saudi Arabia \\ ${ }^{4}$ College of Medicine, King Khalid University, Abha, Saudi Arabia \\ ${ }^{5}$ College of Medicine, Vision Colleges, Riyadh, Saudi Arabia \\ ${ }^{6}$ Primary Healthcare, Althaghar Hospital, Jeddah, Saudi Arabia \\ ${ }^{7}$ Department of Internal Medicine, East Jeddah Hospital, Jeddah, Saudi Arabia
}

Received: 22 December 2021

Accepted: 06 January 2022

\section{*Correspondence:}

Dr. Rahmathulla Safiyul Rahman,

E-mail: safiyul@gmail.com

Copyright: ( ) the author(s), publisher and licensee Medip Academy. This is an open-access article distributed under the terms of the Creative Commons Attribution Non-Commercial License, which permits unrestricted non-commercial use, distribution, and reproduction in any medium, provided the original work is properly cited.

\begin{abstract}
The main intervention to reduce the macro-and microvascular complications of diabetes mellitus (DM) remains to achieve better long-term glycemic control. We have discussed the clinical and economic advantages of using continuous glucose monitoring (CGM) devices for type 1 DM and type 2 DM (T1DM and T2DM) based on data from relevant studies in the literature. Our findings show that using these modalities is associated with remarkable outcomes, including reduced HbA1c levels and enhanced glycemic control among patients with T1DM and T2DM. This can enhance the quality of care and life for diabetic patients and intervene against the development of serious complications and hypoglycemia-related adverse events. The cost of routinely using these devices might seem relatively high. However, the estimated cost benefits are usually higher as they can significantly reduce hospitalization rates due to hypoglycemia and the frequency of diabetic therapy malpractices, which are frequently encountered. However, not many studies have reported these outcomes, indicating the need to conduct future relevant studies.
\end{abstract}

Keywords: Glycemic control, DM, CGM, Management

\section{INTRODUCTION}

Diabetes mellitus (DM) is a highly prevalent disorder and one of the commonest disorders worldwide. The prevalence of DM is also steadily increasing, with a significant impact on the affected patients and communities. It is usually associated with various complications that might affect the affected patient's life. ${ }^{1,2}$ If not deadly, these complications can significantly impair the quality of life of these patients and pose a significant impact on healthcare settings. Therefore, it is essential to reduce the risk of these events and enhance the quality of care for patients with diabetes to improve their life expectancy and reduce the frequency of morbidities and mortality rates. ${ }^{3}$

The main intervention to reduce the macro-and microvascular complications of DM remains to achieve 
better long-term glycemic control. ${ }^{4-6}$ However, evidence indicates that achieving this is usually challenging for T1DM and T2DM patients. Nevertheless, many modalities have been reported for achieving such outcomes and enhancing glycemic control in this context. For instance, CGM devices have been reported with many advantages and enhanced compliance. ${ }^{7}$ In the present literature review, we will discuss the clinical and economic benefits of using CGM devices in achieving better glycemic control based on evidence from the relevant studies in the literature.

\section{LITERATURE REVIEW}

This literature review is based on an extensive literature search in Medline, Cochrane, and EMBASE databases which was performed on $27^{\text {th }}$ December 2021 using the medical subject headings (MeSH) or a combination of all possible related terms, according to the database. To avoid missing potential studies, a further manual search for papers was done through Google Scholar while the reference lists of the initially included papers. Papers discussing clinical and economic benefits of continuous blood glucose monitoring devices were screened for useful information. No limitations were posed on date, language, age of participants, or publication type.

\section{DISCUSSION}

Many studies assessed the current literature's clinical and economic benefits of using CGM devices. Most of these studies indicate the favorable events of using these devices for type I and type II patients. For example, a previous randomized controlled trial (RCT) aimed to assess the clinical efficacy of CGM among adult and children patients with T1DM. They all had adequate control of the disease $(\mathrm{HbA} 1 \mathrm{c}=7-10 \%)$ and received CSII. The authors reported that using CGM devices for 26 weeks was significantly associated with better outcomes regarding all glycemic parameters, including a significant reduction in HbA1c levels. The authors furtherly showed that the reported significant reduction in these patients was more significant than the estimated reduction in patients with SMBG aging $>25$ years $(p<0.001){ }^{8}$ Another analysis of the same patient group also indicated that CGM devices correlated with a numerical decrease in the time spent in hypoglycemia compared to the control group. However, these differences were not statistically significant. On the other hand, the authors demonstrated that there was a significant reduction in HbA1c levels in the patient group and time spent out of range $(\mathrm{p}=0.003){ }^{9}$ Similar favorable outcomes were also reported in the DIAMOND study. The authors compared the outcomes of patients using CGM devices and others receiving diabetic usual care (SMBG $\geq$ four times daily). After 24 weeks from applying these interventions, the authors reported that there was a significant difference regarding the duration of hypoglycemia and HbAlc levels, which were shorter and higher for patients with CGM devices, respectively $(\mathrm{p}=0.002,<0.001){ }^{10}$
Many other investigations also aimed to assess the efficacy of CGM devices for diabetic patients with a history of severe hypoglycemia and impaired awareness of hypoglycemia. For instance, a previous study compared the outcomes of patients using CGM devices and SMBG with CSII and multiple daily injections of insulin. The authors reported that both groups had similar outcomes regarding reductions in the frequency of hypoglycemic events and improvements in hypoglycemia awareness over 24 weeks. ${ }^{11}$ Moreover, another study compared patients using CGM and SMBG after receiving multiple daily injections of insulin or CSII. The included patients were all T1DM and had impaired awareness of hypoglycemia. The authors reported that using CGM devices was significantly associated with a significant reduction in hypoglycemia time, increased normoglycemia time, and reduced severe hypoglycemic events. ${ }^{12}$ Another RCT also compared the efficacy of both CGM and SMBG in T1DM patients receiving multiple daily insulin injections. The authors reported that the incidence of hypoglycemic events was significantly reduced by $72 \%$ among high-risk patients using CGM devices $(p<0.0001) .{ }^{13}$ It has been further shown that CGM devices might also enhance the therapeutic responses and related outcomes of patients receiving different insulin formulations and other antidiabetic modalities. In this context, a previous study aimed to assess the different aspects of glycemic control by using CGM devices among patients receiving two concentrations of insulin glargine. The authors reported that the patients were randomized to receive a dose of 100 or $300 \mathrm{U} / \mathrm{ml}$ of insulin for eight weeks in the morning or evening and vice versa in the following eight weeks. Based on the 24-hour CGM findings, the authors concluded that fewer glucose fluctuations and more consistent glucose readings were significantly associated with administering insulin glargine 300 than $100 \mathrm{U} / \mathrm{ml}$, irrespective of the time of administration. It has also been found that reduced events of confirmed severe nocturnal hypoglycemia were significantly associated with administering 300 than $100 \mathrm{U} / \mathrm{ml}$ of insulin glargine. ${ }^{14}$ Another CGM-based investigation also indicated the clinical efficacy of these modalities in predicting clinical outcomes among patients with a sodium-glucose cotransporter 2 inhibitor. ${ }^{15}$ These findings indicate the clinical efficacy and advantages of using CGM for monitoring glucose levels and related outcomes in diabetic patients.

In addition to the current trials' results, evidence from other studies also indicated that using CGM is also associated with other favorable outcomes. These outcomes might include enhanced quality of life, reduced diabetes-related stress, fear of hypoglycemia, and reduced hypoglycemia and HbA1c levels. ${ }^{16-18}$ In addition, another investigation that included patients with T1DM who began to use CGM devices showed that work absenteeism, hospital stay, and hospitalization due to ketoacidosis and/or hypoglycemia was significantly reduced during one year of follow-up. ${ }^{19}$ However, among 
the different studies in the literature, conclusive evidence is still missing, and the present data is conflicting.

Regarding the use and clinical benefits of CGM devices in monitoring blood glucose levels for patients with T2DM, current evidence indicates that using these devices is associated with enhanced glycemic control among patients receiving multiple daily insulin injections and other antidiabetic modalities. ${ }^{20,21}$ However, the current data is limited by the small number of relevant investigations, indicating the need for future investigations. In this context, a previous RCT investigated these outcomes among T2DM patients receiving various insulin therapy regimens (but no prandial insulin). The follow-up period lasted for 52 weeks, and the authors reported a significant reduction in HbA1c levels in patients with CGM devices than with SMBG. ${ }^{22}$ This has been furtherly indicated by the results from the DIAMOND trial, which showed that using CGM devices was significantly associated with a reduction in HbA1c levels by $1 \%$ more than usual care $(0.6 \%)$ in T2DM patients receiving multiple daily injections of insulin $(\mathrm{p}=0.005)$. However, it should also be noted that the authors reported no significant differences in changes from baseline in insulin dose and time spent in hypoglycemia between the two groups. ${ }^{10}$ In another context, data from the DIAMOND study indicate that there was a significant reduction in baseline HbA1c levels in older T2DM and T1DM patients with either CGM or SMBG $(p<0.001) .^{23}$ Therefore, more recent investigations also aimed to assess the efficacy and benefits of using flash CGM in these patients. For instance, two previous RCTs reported a significant reduction in time spent in hypoglycemia by $43 \%$ and $56 \%$ for adult T2DM patients receiving insulin therapy, and the latter ratio was estimated for adults $\geq 65$ years when using this modality. The authors furtherly reported that the estimated reduction rate for patients with well-controlled T1DM was $38 \% .^{24,25}$ It should be noted that the estimated reductions in all of these occasions were more significant in the CGM than the SMBG group, indicating the clinical efficacy and benefits of using CGM devices.

A previous investigation that included both T1DM and T2DM reported that using flash CGM in these patients was significantly associated with more reduction in HbA1c levels than SMBG. Moreover, it has been shown that more remarkable differences were observed among T1DM patients. ${ }^{26}$ Favorable events were also reported by a previous large investigation, which included 50,000 participants. Using flash CGM approaches, the authors reported a positive correlation between time spent in euglycemia and a negative correlation between time spent in hypo-and hyperglycemia. ${ }^{27}$ On the other hand, another investigation (namely the I HART CGM) reported that among adult patients with T1DM with an increased risk of developing hypoglycemia, there was a significant positive impact over hypoglycemia when switching from flash to real-time CGM. According to the authors of this study, the frequency of time spent on hypoglycemia reduced from $5 \%$ to $0.8 \% .^{28}$ Therefore, these findings indicate that it is important that data collection regarding blood glucose levels should be frequently collected to optimize blood glucose levels and reduce the incidence of hypoglycemia for diabetic patients. However, it should be noted that the economic burden of such approaches is the main barrier and should be considered when drawing such interventions.

The cost-efficacy of using CGM devices has been remarkably variant across the different studies in the literature. However, overall, evidence shows that the annual costs of these devices usually range between 2,500 and 6,000\$, and the estimated cost for buying the flash CMG device is $100 \$$. Moreover, the monthly costs of buying sensor kits for the device usually range between 120 and $200 \$$. It should all be noted that other devices might have higher costs than this, and the cost usually differs between countries based on each manufacturer and economic and political variations. Evidence also indicates that battery replacement usually costs around $600 \$$ per year. ${ }^{29}$ Unfortunately, not many studies in the literature have estimated the cost-efficacy of CGM devices for T2DM patients. Therefore, the frequency of buying these devices and supporting their use by healthcare authorities is questionable and needs further research. However, some studies indicated the cost-efficacy and favorable clinical outcomes in their diabetic populations. For instance, the previous DIAMOND RCT indicated that using CGM devices was significantly associated with remarkable cost-effectiveness during the six months of the trial. ${ }^{30}$ Another study in England also aimed to assess the cost-effectiveness of CMG devices for patients with T1DM. The authors reported that using these devices was significantly associated with net favorable outcomes. Despite the high costs of using these devices, the authors demonstrated that they were remarkably low secondary to the reduced use of insulin pumps, decreased $\mathrm{HbA1c}$ related complications, reduced SMBG strip usage, and decreased hypoglycemia-related costs and adverse events. $^{31}$ On the other hand, another Swedish investigation demonstrated that using flash monitoring devices was more significantly associated with enhanced cost-efficacy than using either CGM or SMBG. ${ }^{32}$ Therefore, it is essential to conduct further investigations to adequately estimate the cost-effectiveness of these modalities and enhance reporting guidelines. Besides, there are some limitations to be considered among the relevant trials in the literature. For instance, it has been shown that most of the included patients in these trials are recruited with high adherence rates to the treatment regimens. However, real-world data show that these rates are significantly lower in T1DM and T2DM patients. Therefore, these differences should be considered, affecting the outcomes and reporting quality. Moreover, there are some barriers to using CGM. These include patient annoyance secondary to interference with daily life, body image issues, insertion pain, frequent alarms, inadequate cost/reimbursement, and limited accuracy of the devices. ${ }^{33}$ Accordingly, further studies are needed to 
overcome these limitations and enhance the quality of these devices and the quality of care of diabetic patients.

\section{CONCLUSION}

We have discussed the clinical and economic advantages of using CGM devices for T1DM and T2DM based on data from relevant studies in the literature. Our findings show that using these modalities is associated with remarkable outcomes, including reduced HbA1c levels and enhanced glycemic control among patients with T1DM and T2DM. This can enhance the quality of care and life for diabetic patients and intervene against the development of serious complications and hypoglycemiarelated adverse events. The cost of routinely using these devices might seem relatively high. However, the estimated cost benefits are usually higher as they can significantly reduce hospitalization rates due to hypoglycemia and the frequency of diabetic therapy malpractices, which are frequently encountered. However, not many studies have reported these outcomes, indicating the need to conduct future relevant studies.

Funding: No funding sources

Conflict of interest: None declared

Ethical approval: Not required

\section{REFERENCES}

1. Gregg EW, Gu Q, Cheng YJ, Narayan KM, Cowie CC. Mortality trends in men and women with diabetes, 1971 to 2000. Ann Internal Med. 2007;147(3):149-55.

2. Gregg EW, Sattar N, Ali MK. The changing face of diabetes complications. Lancet Diabetes Endocrinol. 2016;4(6):537-47.

3. Rubin RR, Peyrot M. Quality of life and diabetes. Diabetes/Metabol Res Rev. 1999;15(3):205-18.

4. Effect of intensive therapy on the development and progression of diabetic nephropathy in the Diabetes Control and Complications Trial. The Diabetes Control and Complications (DCCT) Research Group. Kidney Int. 1995;47(6):1703-20.

5. Adler AI, Stratton IM, Neil HA. Association of systolic blood pressure with macrovascular and microvascular complications of type 2 diabetes (UKPDS 36): prospective observational study. BMJ. 2000;321(7258):412-9.

6. Nathan DM, Cleary PA, Backlund JY. Intensive diabetes treatment and cardiovascular disease in patients with type 1 diabetes. $\mathrm{N}$ Eng $\mathrm{J}$ Med. 2005;353(25):2643-53.

7. Ajjan RA. How Can We Realize the Clinical Benefits of Continuous Glucose Monitoring? Diabetes Technol Therapeutics. 2017;19(S2):S27-36.

8. Tamborlane WV, Beck RW, Bode BW. Continuous glucose monitoring and intensive treatment of type 1 diabetes. N Eng J Med. 2008;359(14):1464-76.

9. Beck RW, Buckingham B, Miller K. Factors predictive of use and of benefit from continuous glucose monitoring in type 1 diabetes. Diabetes Care. 2009;32(11):1947-53.

10. Beck RW, Riddlesworth T, Ruedy K. Effect of Continuous Glucose Monitoring on Glycemic Control in Adults With Type 1 Diabetes Using Insulin Injections: The DIAMOND Randomized Clinical Trial. JAMA. 2017;317(4):371-8.

11. Little SA, Leelarathna L, Walkinshaw E. Recovery of hypoglycemia awareness in long-standing type 1 diabetes: a multicenter $2 \times 2$ factorial randomized controlled trial comparing insulin pump with multiple daily injections and continuous with conventional glucose self-monitoring (HypoCOMPaSS). Diabetes Care. 2014;37(8):211422.

12. Van Beers CA, DeVries JH, Kleijer SJ, et al. Continuous glucose monitoring for patients with type 1 diabetes and impaired awareness of hypoglycaemia (IN CONTROL): a randomised, open-label, crossover trial. Lancet Diabetes Endocrinol. 2016;4(11):893-902.

13. Heinemann L, Freckmann G, Ehrmann D. Real-time continuous glucose monitoring in adults with type 1 diabetes and impaired hypoglycaemia awareness or severe hypoglycaemia treated with multiple daily insulin injections (HypoDE): a multicentre, randomised controlled trial. Lancet (London, England). 2018;391(10128):1367-77.

14. Bergenstal RM, Bailey TS, Rodbard D. Comparison of Insulin Glargine 300 Units/mL and 100 Units/mL in Adults With Type 1 Diabetes: Continuous Glucose Monitoring Profiles and Variability Using Morning or Evening Injections. Diabetes Care. 2017;40(4):554-60.

15. Argento NB, Nakamura K. Glycemic effects of SGLT-2 inhibitor canagliflozin in type 1 diabetes patients using the dexcom G4 platinum CGM. Endocrine Practice. 2016;22(3):315-22.

16. Patton SR, Clements MA. Psychological Reactions Associated With Continuous Glucose Monitoring in Youth. J Diabetes Sci Technol. 2016;10(3):656-61.

17. Beck RW, Lawrence JM, Laffel L. Quality-of-life measures in children and adults with type 1 diabetes: Juvenile Diabetes Research Foundation Continuous Glucose Monitoring randomized trial. Diabetes Care. 2010;33(10):2175-7.

18. Lind M, Polonsky W, Hirsch IB. Continuous Glucose Monitoring vs Conventional Therapy for Glycemic Control in Adults With Type 1 Diabetes Treated With Multiple Daily Insulin Injections: The GOLD Randomized Clinical Trial. JAMA. 2017;317(4):37987.

19. Charleer S, Mathieu C, Nobels F. Effect of Continuous Glucose Monitoring on Glycemic Control, Acute Admissions, and Quality of Life: A Real-World Study. J Clin Endocrinol Metabol. 2018;103(3):1224-32.

20. Vigersky RA, Fonda SJ, Chellappa M, Walker MS, Ehrhardt NM. Short- and long-term effects of real- 
time continuous glucose monitoring in patients with type 2 diabetes. Diabetes Care. 2012;35(1):32-8.

21. Beck RW, Riddlesworth TD, Ruedy K. Continuous Glucose Monitoring Versus Usual Care in Patients With Type 2 Diabetes Receiving Multiple Daily Insulin Injections: A Randomized Trial. Ann Internal Med. 2017;167(6):365-74.

22. Ehrhardt NM, Chellappa M, Walker MS, Fonda SJ, Vigersky RA. The effect of real-time continuous glucose monitoring on glycemic control in patients with type 2 diabetes mellitus. J Diabetes Sci Technol. 2011;5(3):668-75.

23. Ruedy KJ, Parkin CG, Riddlesworth TD, Graham C. Continuous Glucose Monitoring in Older Adults With Type 1 and Type 2 Diabetes Using Multiple Daily Injections of Insulin: Results From the DIAMOND Trial. J Diabetes Sci Technol. 2017;11(6):1138-46.

24. Haak T, Hanaire H, Ajjan R, Hermanns N, Riveline JP, Rayman G. Flash Glucose-Sensing Technology as a Replacement for Blood Glucose Monitoring for the Management of Insulin-Treated Type 2 Diabetes: a Multicenter, Open-Label Randomized Controlled Trial. Diabetes Therapy. 2017;8(1):55-73.

25. Bolinder $J$, Antuna $R$, Geelhoed-Duijvestijn $P$, Kröger J, Weitgasser R. Novel glucose-sensing technology and hypoglycaemia in type 1 diabetes: a multicentre, non-masked, randomised controlled trial. Lancet (London, England). 2016;388(10057):2254-63.

26. Anjana RM, Kesavadev J, Neeta D. A Multicenter Real-Life Study on the Effect of Flash Glucose Monitoring on Glycemic Control in Patients with Type 1 and Type 2 Diabetes. Diabetes Technol Therapeutics. 2017;19(9):533-40.

27. Dunn TC, Xu Y, Hayter G, Ajjan RA. Real-world flash glucose monitoring patterns and associations between self-monitoring frequency and glycaemic measures: A European analysis of over 60 million glucose tests. Diabetes Res Clin Pract. 2018;137:3746.
28. Reddy M, Jugnee N, Anantharaja S, Oliver N. Switching from Flash Glucose Monitoring to Continuous Glucose Monitoring on Hypoglycemia in Adults with Type 1 Diabetes at High Hypoglycemia Risk: The Extension Phase of the I HART CGM Study. Diabetes Technol Therapeutics. 2018;20(11):751-7.

29. Robertson SL, Shaughnessy AF, Slawson DC. Continuous Glucose Monitoring in Type 2 Diabetes Is Not Ready for Widespread Adoption. Am Family Physician. 2020;101(11):646.

30. Wan W, Skandari MR, Minc A. Cost-effectiveness of Continuous Glucose Monitoring for Adults With Type 1 Diabetes Compared With Self-Monitoring of Blood Glucose: The DIAMOND Randomized Trial. Diabetes Care. 2018;41(6):1227-34.

31. Chaugule S, Oliver N, Klinkenbijl B, Graham C. An Economic Evaluation of Continuous Glucose Monitoring for People with Type 1 Diabetes and Impaired Awareness of Hypoglycaemia within North West London Clinical Commissioning Groups in England. European Endocrinol. 2017;13(2):81-5.

32. Blissett DB, Attvall S, Hellmund RA. Analysis Estimating the Potential Cost Impact of Utilizing Flash Glucose Monitoring with Optional Alarms in Swedish Adults with Diabetes with Impaired Awareness of Hypoglycaemia, Using Intensive Insulin. Diabetes Therapy. 2021;12(8):2179-93.

33. Ajjan R, Slattery D, Wright E. Continuous Glucose Monitoring: A Brief Review for Primary Care Practitioners. Adv Therapy. 2019;36(3):579-96.

Cite this article as: Rahman RS, Salamah HE, Alshair FM, Alsawadi AA, Alghamdi OM, Alalasi $\mathrm{AB}$ et al. Clinical and economic benefits of continuous blood glucose monitoring devices. Int J Community Med Public Health 2022;9:1128-32. 